2013-09-15

\title{
A novel bioassay using root re-growth in Lemna.
}

\section{Park, A}

http://hdl.handle.net/10026.1/3921

10.1016/j.aquatox.2013.06.004

Aquat Toxicol

All content in PEARL is protected by copyright law. Author manuscripts are made available in accordance with publisher policies. Please cite only the published version using the details provided on the item record or document. In the absence of an open licence (e.g. Creative Commons), permissions for further reuse of content should be sought from the publisher or author. 


\title{
A novel bioassay using root re-growth in Lemna
}

\author{
Areum Park ${ }^{\mathrm{a}}$, Youn-Jung Kim ${ }^{\mathrm{b}}$, Eun-Mi Choi ${ }^{\mathrm{c}}$, Murray T. Brown ${ }^{\mathrm{d}}$, Taejun Han ${ }^{\mathrm{b}, \mathrm{e}, *}$ \\ a Division of Life Science, Incheon National University, 12-1 Songdo-dong, Yeonsu-gu, Incheon 406-840, Republic of Korea \\ ${ }^{\mathrm{b}}$ Department of Marine Science, Incheon National University, 12-1 Songdo-dong, Yeonsu-gu, Incheon 406-840, Republic of Korea \\ ${ }^{\mathrm{c}}$ Department of Chemistry, Incheon National University, 12-1 Songdo-dong, Yeonsu-gu, Incheon 406-840, Republic of Korea \\ d School of Marine Science E' Engineering, Plymouth University, Plymouth, Devon PL4 8AA, United Kingdom \\ e Institute of Green Environmental Research, Incheon National University, 7-46 Songdo-dong, Yeonsu-gu, Incheon 406-840, Republic of Korea
}

\section{A R T I C L E I N F O}

\section{Article history:}

Received 3 January 2013

Received in revised form 3 June 2013

Accepted 7 June 2013

\section{Keywords:}

Lemna gibba

L. minor

L. paucicostata

Metals

Root growth

\begin{abstract}
A B S T R A C T
A new phytotoxicity test method based on root elongation of three Lemna species (Lemna gibba, L. minor, and $L$. paucicostata) has been developed. Tests with aquatic plants have, typically, favored measurements on fronds (e.g. frond number, area, biomass) rather than on roots, due, in part, to issues associated with handling fragile roots and the time-consuming procedures of selecting roots with identical root lengths. The present method differs in that roots were excised prior to exposure with subsequent measurements on newly developed roots. Results show that there were species-specific difference in sensitivity to the five metals tested $(\mathrm{Ag}, \mathrm{Cd}, \mathrm{Cr}, \mathrm{Cu}$ and $\mathrm{Hg})$, with $\mathrm{Ag}$ being the most toxic $\left(\mathrm{EC}_{50}=5.3-37.6 \mu \mathrm{L} \mathrm{L}^{-1}\right)$ to all three species, and $\mathrm{Cr}$ the least toxic for $L$. gibba and L. minor (1148.3 and $341.8 \mu \mathrm{g} \mathrm{L}^{-1}$, respectively) and $\mathrm{Cu}$ for L. paucicostata $\left(470.4 \mu \mathrm{g} \mathrm{L}^{-1}\right)$. Direct comparisons were made with measurements of frond area, which were found to be less sensitive. More generally, root re-growth was shown to reflect the toxic responses of all three Lemna species to these five important metals.

The root growth bioassay differs from three internationally standardized methods (ISO, OCED and US $\mathrm{EPA}$ ) in that it is completed in $48 \mathrm{~h}$, the required volume of test solutions is only $3 \mathrm{ml}$ and non-axenic plants are used. Our results show that the Lemna root method is a simple, rapid, cost-effective, sensitive and precise bioassay to assess the toxic risks of metals and has practical application for monitoring municipal and industrial waste waters where metals are common constituents.
\end{abstract}

(ㄷ) 2013 Elsevier B.V. All rights reserved.

\section{Introduction}

The global importance of detecting toxicants in the environment and assessing their impacts on biota has resulted in the development and adoption of a range of methodologies. Direct chemical analysis has several drawbacks that include the complexity of the procedures for preparing samples, the need for expensive analytical equipment and interference from secondary pollutants during analysis. In addition, this approach does not take account of temporal changes in exposure or the interactive effects of pollutants, nor does it provide ecologically significant information (Kumar and Han, 2010). To compensate for these limitations, various biological assays have been developed, including some that employ aquatic plants, in order to provide information on pollutant-induced toxic effects that can be used to assess environmental risks (Eullaffroy and Vernet, 2003).

\footnotetext{
* Corresponding author at: Department of Marine Science, Incheon National University, 12-1 Songdo-dong, Yeonsu-gu, Incheon 406-840, Republic of Korea. Tel.: +82 32 8505711; fax: +82328505717.

E-mail address: hanalgae@hanmail.net (T. Han).
}

Aquatic vascular plants belonging to the genus Lemna have gained broad acceptance as bioassays in ecotoxicological research. Lemna spp. have many advantages as test organisms, including their simple structure and small size, allowing for small volumes of sample toxicants to be used (Li and Xiong, 2004; Kumar and Han, 2010), rapid rates of growth (a doubling time of 1-4 d), ease of culture and handling, high degree of homogeneity such that most clones are morphologically similar (Lahive et al., 2011), and sensitivity to a wide range of pollutants (Hillman, 1961; Wang and Williams, 1990; Christen and Theuer, 1996). Moreover, Lemna plants are appealing test organisms due to their ecological importance as primary producers and their wide geographical occurrence (Drost et al., 2007). Among Lemna spp., L. gibba and $L$ minor have been most extensively used in phytotoxicity testing and there are several standard methods which have been adopted by major international standardization agencies e.g. U.S. Environmental Protection Agency (USEPA, 1996), Organization for Economic Cooperation and Development (OECD, 2002), and International Standardization Organization (ISO, 2005).

To ensure thorough evaluation of the risks posed by pollutants for environmental and human health, the test methods employed should be sensitive, rapid, simple and of ecological relevance. 
Therefore, a technique that can assess toxicity more rapidly but without loss of sensitivity would be a valuable asset. In the case of Lemna, bioassays using traditional endpoints such as number of fronds, wet or dry biomass and growth rates of fronds require standard exposure durations of at least $7 \mathrm{~d}$ to detect toxicity. Tests based on root elongation are some of the most widely used phytotoxicity methodologies for terrestrial angiosperms (Wang, 1991) because of their simplicity and rapidity (Munzuroglu and Geckil, 2002). However, despite reports that roots of L. minor are highly sensitive to environmental stressors (Panda and Upadhyay, 2003) and that they play important ecological roles by providing stability and facilitate dispersal there have been few studies incorporating root elongation as a test endpoint (Davis, 1981). This may be due, in part, to the fragility of the roots, which makes handling of roots for measurements of length difficult (Davis, 1981). It can also be impractical to obtain sufficient numbers of individual plants with identical root lengths for initiating tests.

The aim of this study was to develop a new root elongation test method using three Lemna species (L. gibba, L. minor, and L. paucicostata) and to evaluate the sensitivity and consistency of the method upon exposure to 5 different essential and non-essential metals ( $\mathrm{Ag}, \mathrm{Cd}, \mathrm{Cr}, \mathrm{Cu}$ and $\mathrm{Hg}$ ). Metals derived from anthropogenic activities and released into the aquatic environments are not degraded, and represent a serious source of environmental pollution for plant function (Teisseire and Vernet, 2000; Prasad et al., 2001; Zhou et al., 2009) and to higher trophic organisms via bioaccumulation through food chains (Li and Xiong, 2004; Drost et al., 2007; Khellaf and Zerdaoui, 2009).

The new test method presented in this study differs from the traditional procedures by removing all roots from fronds prior to exposure to toxicants and measuring growth of newly developed roots during the exposure period of $48 \mathrm{~h}$.

\section{Materials and methods}

\subsection{Sample collection and maintenance}

Lemna gibba (CPCC 310) and L. minor (CPCC 490) were obtained from the Canadian Phycological Culture Center. L. paucicostata was collected from an artificial fresh water pond located in a botanical garden in Gwang-ju, Republic of Korea (35.09 N, 125.54 E). Maintenance and cultivation of fronds were carried out under continuous white light $\left(20-30 \mu \mathrm{mol}\right.$ photons $\left.\mathrm{m}^{-2} \mathrm{~s}^{-1}\right)$ at $20^{\circ} \mathrm{C}$ in $10 \mathrm{ml}$ plastic tank with Steinberg growth medium (Steinberg, 1946) under static conditions. Low fluorescent lighting was employed to ensure less likelihood of contamination. The $\mathrm{pH}$ of the medium was adjusted to $7 \pm 0.2$ with solutions of hydrochloric acid $(\mathrm{HCl})$ or sodium hydroxide $(\mathrm{NaOH})$ at strengths $\leq 1 \mathrm{M}$.

\subsection{Experimental procedures}

To determine the optimal conditions for frond and root growth of each species, healthy green plants, each with two fronds, were selected and their roots excised using stainless steel scissors. Individual rootless plants were then placed in each cell of a 24-well plate $(85.4 \mathrm{~mm} \times 127.6 \mathrm{~mm}$; well dimension $15.6 \mathrm{~mm}$ diameter, SPL, Korea), and exposed for $48 \mathrm{~h}$ to various levels of environmental factors: photon irradiance (50-200 $\mu \mathrm{mol}$ photons $\left.\mathrm{m}^{-2} \mathrm{~s}^{-1}\right)$, temperature $\left(10-35^{\circ} \mathrm{C}\right)$ and $\mathrm{pH}(3-10)$. While testing each single factor, the other environmental conditions were maintained optimally: photon irradiance at $150 \mu \mathrm{mol}$ photons $\mathrm{m}^{-2} \mathrm{~s}^{-1}$, temperature at $30^{\circ} \mathrm{C}$ and $\mathrm{pH}$ 7. The growth medium was not replaced during the incubation period. After 48-h incubation, all plants were harvested and the longest root lengths (each frond generally has 1-2 roots) were measured using an image analyzer (Moticam 2500, Ted Pella
Table 1

Final concentration range used for testing toxicity of metals with three species of Lemna.

\begin{tabular}{lrrr}
\hline $\begin{array}{l}\text { Metal concentrations } \\
\left(\mu \mathrm{g} \mathrm{L}^{-1}\right)\end{array}$ & L. gibba & L. minor & L. paucicostata \\
\hline $\mathrm{Ag}$ & $6.25-100$ & $3.125-100$ & $7.8125-125$ \\
$\mathrm{Cd}$ & $31.25-1000$ & $31.25-2000$ & $19.53-625$ \\
$\mathrm{Cr}$ & $93.75-3000$ & $46.875-3000$ & $156.25-2500$ \\
$\mathrm{Cu}$ & $125-4000$ & $62.5-4000$ & $156.25-5000$ \\
$\mathrm{Hg}$ & $15.625-2000$ & $15.625-1000$ & $78.125-2500$ \\
\hline
\end{tabular}

Inc., USA). For comparison, frond areas were also measured with the same instrument.

\subsection{Toxicity test}

Toxicity tests were carried out in a growth chamber under the optimal conditions established from the experiment described above, i.e. continuous light of $150 \mu \mathrm{mol}$ photons $\mathrm{m}^{-2} \mathrm{~s}^{-1}, \mathrm{pH} 7$ and $30^{\circ} \mathrm{C}$. The test was a static type, with no renewal of solutions during the period of exposure. The test vessel was a 24 -well plastic plate with $3 \mathrm{ml}$ of test solution added to each well. Individual rootless plants were added to cells of the plate; there were 4 plants per concentration, 6 concentrations of metal per plate and three replicate plates were exposed for $48 \mathrm{~h}$. A different range of metal concentrations depending on metal were made by diluting the original stock solutions of $\mathrm{AgNO}_{3}$ (CAS no. 7440-22-4, SHOWA, Japan), $\mathrm{CdSO}_{4}$ (CAS no. 7440-43-9, SHOWA, Japan), $\mathrm{K}_{2} \mathrm{Cr}_{2} \mathrm{O}_{7}$ (CAS no. 7789-006, SHOWA, Japan), $\mathrm{CuSO}_{4}$ (CAS no. 7440-50-8, SHOWA, Japan), and $\mathrm{HgCl}_{2}$ (CAS no. 7439-97-6, SHOWA, Japan) with Steinberg medium. Final metal concentration range used is present in Table 1. In carrying out the experimental procedures, a fully randomized design was used to account for any variability in environmental conditions within the culture cabinet.

\subsection{Statistical analysis}

Analysis of variance was performed to confirm significant differences in response. Multiple comparison tests by the least significance difference (LSD) were then carried out to find significant differences from the controls and between treatments. Results are reported as $\mathrm{EC}_{50} \mathrm{~s}$ (the effective concentrations at which $50 \%$ inhibition occurs) with $95 \%$ confidence intervals estimated by the linear interpolated method (ToxCalc 5.0, Tidepool Science, CA, USA).

\section{Results and discussion}

Several environmental factors can influence the toxicity of contaminants to Lemna species, and so standardization of the toxicity test was established by determining the optimal conditions for root and frond growth of all three Lemna species. The growth of three species incubated for $48 \mathrm{~h}$ under different photon irradiances, $\mathrm{pH}$ and temperatures is shown in Figs. 1-3. In all the tested species, growth of roots and fronds was saturated at irradiances higher than $50 \mu \mathrm{mol}$ photons $\mathrm{m}^{-2} \mathrm{~s}^{-1}$ while there was no evidence of growth in the dark. Lemna is known to be a low light plant, with an adequate requirement of photon irradiances for rapid growth at 60-100 $\mu \mathrm{mol}$ photons $\mathrm{m}^{-2} \mathrm{~s}^{-1}$ (Greenberg et al., 1992). The maximal growth of the three Lemna species occurred at irradiances higher than $50 \mu \mathrm{mol}$ photons $\mathrm{m}^{-2} \mathrm{~s}^{-1}$ for L. gibba and L. paucicostata and $100 \mu \mathrm{mol}$ photons $\mathrm{m}^{-2} \mathrm{~s}^{-1}$ for $L$. minor. Therefore, quantum irradiance was set at $150 \mu \mathrm{mol}$ photons $\mathrm{m}^{-2} \mathrm{~s}^{-1}$ for the toxicity tests. As shown in Table 2, it is notable that Lemna tests have been conducted at photon irradiances of 40-129 $\mu \mathrm{mol}$ photons $\mathrm{m}^{-2} \mathrm{~s}^{-1}$.

Optimum growth temperatures for Lemna are known to be between 25 and $31^{\circ} \mathrm{C}$ and the temperature minima and maxima are 
Table 2

List of metal toxicity tests using various endpoints in three Lemna species (L. gibba, L. minor and L. paucicostata).

\begin{tabular}{|c|c|c|c|c|c|c|c|c|c|c|c|}
\hline Toxicant & Test organism & End-point & $\begin{array}{l}\text { Exposure } \\
\text { time }\end{array}$ & $\begin{array}{l}\mathrm{EC}_{50} \\
\left(\mu \mathrm{L}^{-1}\right)\end{array}$ & $\begin{array}{l}\text { Test } \\
\text { volume } \\
(\mathrm{ml})\end{array}$ & Medium & $\begin{array}{l}\text { Light } \\
\left(\mu \mathrm{mol} \mathrm{m}^{-2} \mathrm{~s}^{-1}\right)\end{array}$ & $\begin{array}{l}\text { Light } \\
\text { duration } \\
\text { (h) }\end{array}$ & $\begin{array}{l}\text { Temperature } \\
\left({ }^{\circ} \mathrm{C}\right)\end{array}$ & $\mathrm{pH}$ & References \\
\hline $\mathrm{Ag}$ & Lemnagibba & $\mathrm{RL}$ & $2 \mathrm{~d}$ & $\begin{array}{l}27.6 \\
(21.3-34.2)\end{array}$ & 3 & Steinberg & $150 \pm 10$ & 24 & $30 \pm 1$ & 7 & This study \\
\hline $\mathrm{AgNO}_{3}$ & L. minor & $\begin{array}{l}\text { FN } \\
\text { FW } \\
\text { DW } \\
\text { CHL } \\
\text { CAR }\end{array}$ & $7 d$ & $\begin{array}{l}81(66-98) \\
30(24-37) \\
31(26-37) \\
37(17-65) \\
24(18-31)\end{array}$ & 300 & Steinberg & $100 \pm 15$ & 24 & $25 \pm 1$ & 5.5 & $\begin{array}{l}\text { Naumann et al. } \\
\text { (2007) }\end{array}$ \\
\hline $\mathrm{AgNO}_{3}$ & L. minor & $\mathrm{FAB}$ & $7 \mathrm{~h}$ & $\begin{array}{l}78 \\
(56-108)\end{array}$ & & Steinberg & $100 \pm 15$ & 24 & $25 \pm 1$ & 5.5 & $\begin{array}{l}\text { Topp et al. } \\
\text { (2011) }\end{array}$ \\
\hline $\mathrm{AgNP}_{1}$ & L. minor & $\begin{array}{l}\text { FN } \\
\text { DW } \\
\text { FN } \\
\text { DW }\end{array}$ & $\begin{array}{l}7 \mathrm{~d} \\
14 \mathrm{~d}\end{array}$ & $\begin{array}{l}140 \\
640 \\
38 \\
19\end{array}$ & & SIS & & & $24 \pm 2$ & $5.5 \pm 1.4$ & $\begin{array}{l}\text { Gubbins et al. } \\
\text { (2011) }\end{array}$ \\
\hline $\mathrm{AgNP}_{2}$ & L. minor & $\begin{array}{l}\text { FN } \\
\text { DW } \\
\text { FN } \\
\text { DW }\end{array}$ & $\begin{array}{l}7 \mathrm{~d} \\
14 \mathrm{~d}\end{array}$ & $\begin{array}{l}125 \\
62 \\
43 \\
19\end{array}$ & & & & & & & \\
\hline $\mathrm{Ag}$ & L. minor & RL & $2 \mathrm{~d}$ & $\begin{array}{l}5.3 \\
(2.6-23.1)\end{array}$ & 3 & Steinberg & $150 \pm 10$ & 24 & $30 \pm 1$ & 7 & This study \\
\hline $\mathrm{AgNO}_{3}$ & L. paucicostata & FN & $7 d$ & $100-1000$ & 25 & $\begin{array}{l}\text { Bonner- } \\
\text { Devirian }\end{array}$ & 120 & 24 & $25 \pm 1$ & $4.1-7.1$ & $\begin{array}{l}\text { Nasu and } \\
\text { Kugimoto } \\
\text { (1981) }\end{array}$ \\
\hline $\mathrm{Ag}$ & L. paucicostata & $\mathrm{RL}$ & $2 \mathrm{~d}$ & $\begin{array}{l}37.6 \\
(28.6-44.3)\end{array}$ & 3 & Steinberg & $150 \pm 10$ & 24 & $30 \pm 1$ & 7 & This study \\
\hline $\mathrm{Cd}$ & L. gibba & $\mathrm{RL}$ & $2 \mathrm{~d}$ & $\begin{array}{l}363.5 \\
(257.4-407.6)\end{array}$ & 3 & Steinberg & $150 \pm 10$ & 24 & $30 \pm 1$ & 7 & This study \\
\hline $\mathrm{Cd}$ & L. minor & FN & $4 \mathrm{~d}$ & 200 & 200 & $\begin{array}{l}\text { Plant } \\
\text { nutrition } \\
\text { Solution } \\
\text { (Standard } \\
\text { Methods } \\
1980 \text { ) }\end{array}$ & & & $27 \pm 2$ & 7.5 & Wang (1986) \\
\hline $\mathrm{CdCl}_{2}$ & L. minor & $\begin{array}{l}\text { FN } \\
\text { FW } \\
\text { DW } \\
\text { CHL } \\
\text { CAR }\end{array}$ & $7 d$ & $\begin{array}{l}323 \\
(232-450) \\
110 \\
(72-189) \\
241 \\
(151-384) \\
102 \\
(86-125) \\
92 \\
(75-112)\end{array}$ & 300 & Steinberg & $100 \pm 15$ & 24 & $25 \pm 1$ & 5.5 & $\begin{array}{l}\text { Naumann et al. } \\
\text { (2007) }\end{array}$ \\
\hline $\mathrm{CdSO}_{4}$ & L. minor & FN & & 213 & 150 & Steinberg & $85-125$ & 24 & $25 \pm 2$ & $5.5 \pm 0.2$ & $\begin{array}{l}\text { Drost et al. } \\
\text { (2007) }\end{array}$ \\
\hline $3 \mathrm{CdSO}_{4} \cdot 8 \mathrm{H}_{2} \mathrm{O}$ & L. minor & FN & & 2500 & 200 & $\begin{array}{l}\text { Duckweed } \\
\text { nutrient } \\
\text { solution }\end{array}$ & 42 & 16 & $25 \pm 1$ & 5 & Ma et al. (2007) \\
\hline
\end{tabular}


Table 2 (Continued)

\begin{tabular}{|c|c|c|c|c|c|c|c|c|c|c|c|}
\hline Toxicant & Test organism & End-point & $\begin{array}{l}\text { Exposure } \\
\text { time }\end{array}$ & $\begin{array}{l}\mathrm{EC}_{50} \\
\left(\mu \mathrm{gL}^{-1}\right)\end{array}$ & $\begin{array}{l}\begin{array}{l}\text { Test } \\
\text { volume } \\
(\mathrm{ml})\end{array} \\
\end{array}$ & Medium & $\begin{array}{l}\text { Light } \\
\left(\mu \mathrm{mol} \mathrm{m}^{-2} \mathrm{~s}^{-1}\right)\end{array}$ & $\begin{array}{l}\text { Light } \\
\text { duration } \\
\text { (h) }\end{array}$ & $\begin{array}{l}\text { Temperature } \\
\left({ }^{\circ} \mathrm{C}\right)\end{array}$ & $\mathrm{pH}$ & References \\
\hline $\mathrm{Cd}$ & L. minor & $\mathrm{FA}$ & $4 \mathrm{~d}$ & 910 & 100 & $\begin{array}{l}\text { 1/4 Col̈c \& Leisant } \\
\text { (1973) }\end{array}$ & 101 & 16 & 22 & $6.1 \pm 0.1$ & $\begin{array}{l}\text { Khellaf and Zerdaoui } \\
\text { (2009) }\end{array}$ \\
\hline $\mathrm{Cd}$ & L. minor & RL & $2 \mathrm{~d}$ & $\begin{array}{l}226.4 \\
(200.7-248.5)\end{array}$ & 3 & Steinberg & $150 \pm 10$ & 24 & $30 \pm 1$ & 7 & This study \\
\hline $\mathrm{CdCl}_{2} \cdot 2 \frac{1}{2} \mathrm{H}_{2} \mathrm{O}$ & L. paucicostata & FN & $7 d$ & $20-50$ & 25 & Bonner-Devirian & 120 & 24 & $25 \pm 1$ & $4.1-7.1$ & $\begin{array}{l}\text { Nasu and Kugimoto } \\
\text { (1981) }\end{array}$ \\
\hline $\mathrm{Cd}$ & L. paucicostata & RL & $2 \mathrm{~d}$ & $\begin{array}{l}77.0 \\
(61.1-118.1)\end{array}$ & 3 & Steinberg & $150 \pm 10$ & 24 & $30 \pm 1$ & 7 & This study \\
\hline $\mathrm{K}_{2} \mathrm{Cr}_{2} \mathrm{O}_{7}$ & L. gibba & $\begin{array}{l}\text { No plants } \\
\text { FN } \\
\text { DW }\end{array}$ & $7 d$ & $\begin{array}{l}49,000 \\
(12,000-85,000) \\
45,000 \\
(16,000-75,000) \\
48,000 \\
(17,000-79,000)\end{array}$ & 100 & Hoagland & 129 & 24 & $25 \pm 2$ & $4.6-5.4$ & Cowgill et al. (1991) \\
\hline $\mathrm{Cr}(\mathrm{VI})$ & L. gibba & $\mathrm{RL}$ & $2 \mathrm{~d}$ & $\begin{array}{l}1148.3 \\
(276.1-1403.6)\end{array}$ & 3 & Steinberg & $150 \pm 10$ & 24 & $30 \pm 1$ & 7 & This study \\
\hline $\begin{array}{l}\mathrm{K}_{2} \mathrm{Cr}_{2} \mathrm{O}_{7} \\
\mathrm{Cr}(\mathrm{VI})\end{array}$ & $\begin{array}{l}\text { L. minor } \\
\text { L. minor }\end{array}$ & $\begin{array}{l}\text { FN } \\
\text { FN }\end{array}$ & $\begin{array}{l}14 \mathrm{~d} \\
4 \mathrm{~d}\end{array}$ & $\begin{array}{l}5000 \\
35,000\end{array}$ & - & $\begin{array}{l}\text { Btistol } \\
\text { Plant nutrition } \\
\text { solution (Standard } \\
\text { Methods 1980) }\end{array}$ & 40 & 12 & $\begin{array}{l}21 \\
27 \pm 2\end{array}$ & $\begin{array}{l}7.2 \\
7.5\end{array}$ & $\begin{array}{l}\text { Mangi et al. (1978) } \\
\text { Wang (1986) }\end{array}$ \\
\hline $\mathrm{K}_{2} \mathrm{Cr}_{2} \mathrm{O}_{7}$ & L. minor & $\begin{array}{l}\text { No plants } \\
\text { FN } \\
\text { DW }\end{array}$ & $7 d$ & $\begin{array}{l}40,000 \\
(8000-204,000) \\
30,000 \\
(6000-151,000) \\
28,000 \\
(7000-106,000)\end{array}$ & 100 & Hoagland & 108 & 24 & $25 \pm 2$ & $4.6-5.4$ & Cowgill et al. (1991) \\
\hline $\mathrm{Na}_{2} \mathrm{CrO}_{4} \cdot 4 \mathrm{H}_{2} \mathrm{O}$ & L. minor & $\begin{array}{l}\text { FN } \\
\text { FW } \\
\text { DW } \\
\text { CHL } \\
\text { CAR }\end{array}$ & $7 d$ & $\begin{array}{l}2300 \\
(870-8500) \\
584 \\
(33-1060) \\
2300 \\
(870-8500) \\
160 \\
(30-2770) \\
80 \\
(10-6620)\end{array}$ & 300 & Steinberg & $100 \pm 15$ & 24 & $25 \pm 1$ & 5.5 & Naumann et al. (2007) \\
\hline $\mathrm{Cr}(\mathrm{VI})$ & L. minor & $\mathrm{RL}$ & $2 \mathrm{~d}$ & $\begin{array}{l}341.8 \\
(163.0-559.7)\end{array}$ & 3 & Steinberg & $150 \pm 10$ & 24 & $30 \pm 1$ & 7 & This study \\
\hline $\mathrm{K}_{2} \mathrm{Cr}_{2} \mathrm{O}_{7}$ & L. paucicostata & FN & $7 d$ & $>1000$ & 25 & Bonner-Devirian & 120 & 24 & $25 \pm 1$ & $4.1-7.1$ & $\begin{array}{l}\text { Nasu and Kugimoto } \\
\text { (1981) }\end{array}$ \\
\hline $\mathrm{Cr}(\mathrm{VI})$ & L. paucicostata & RL & $2 \mathrm{~d}$ & $\begin{array}{l}237.9 \\
(195.0-280.5)\end{array}$ & 3 & Steinberg & $150 \pm 10$ & 24 & $30 \pm 1$ & 7 & This study \\
\hline $\mathrm{CuSO}_{4} \cdot 5 \mathrm{H}_{2} \mathrm{O}$ & L. gibba & FA & $4 \mathrm{~d}$ & 450 & 100 & $\begin{array}{l}\text { 1/4 Col̈c\& Leisant } \\
\text { (1973) }\end{array}$ & - & 12 & 21 & 6 & $\begin{array}{l}\text { Khellaf and Zerdaoui } \\
\text { (2010) }\end{array}$ \\
\hline $\mathrm{Cu}$ & L. gibba & $\mathrm{RL}$ & $2 \mathrm{~d}$ & $\begin{array}{l}310.0 \\
(236.7-391.3)\end{array}$ & 3 & Steinberg & $150 \pm 10$ & 24 & $30 \pm 1$ & 7 & This study \\
\hline $\mathrm{Cu}$ & L. minor & FN & $4 \mathrm{~d}$ & 1100 & 200 & $\begin{array}{l}\text { Plant nutrition } \\
\text { solution (Standard } \\
\text { Methods 1980) }\end{array}$ & & & $27 \pm 2$ & 7.5 & Wang (1986) \\
\hline
\end{tabular}




\begin{tabular}{|c|c|c|c|c|c|c|c|c|c|c|c|}
\hline Toxicant & Test organism & End-point & $\begin{array}{l}\text { Exposure } \\
\text { time }\end{array}$ & $\begin{array}{l}\mathrm{EC}_{50} \\
\left(\mu \mathrm{L}^{-1}\right)\end{array}$ & $\begin{array}{l}\text { Test } \\
\text { volume } \\
(\mathrm{ml})\end{array}$ & Medium & $\begin{array}{l}\text { Light } \\
\left(\mu \mathrm{mol} \mathrm{m}^{-2} \mathrm{~s}^{-1}\right)\end{array}$ & $\begin{array}{l}\text { Light } \\
\text { duration } \\
\text { (h) }\end{array}$ & $\begin{array}{l}\text { Temperature } \\
\left({ }^{\circ} \mathrm{C}\right)\end{array}$ & $\mathrm{pH}$ & References \\
\hline $\mathrm{CuSO}_{4} \cdot 5 \mathrm{H}_{2} \mathrm{O}$ & L. minor & CHL & $7 d$ & $\begin{array}{l}160 \\
(110-210) \\
160 \\
(120-200)\end{array}$ & 50 & $\begin{array}{l}\text { Inorganic } \\
\text { prowth } \\
\text { medium } \\
\text { (Chollet } \\
\text { 1993) }\end{array}$ & $40 \pm 2$ & 24 & $25 \pm 1$ & 6.5 & $\begin{array}{l}\text { Teisseire et al. } \\
\text { (1998) }\end{array}$ \\
\hline $\mathrm{CuCl}_{2}$ & L. minor & FN & $7 d$ & 616 & 150 & Steinberg & $85-125$ & 24 & $25 \pm 2$ & $5.5 \pm 0.2$ & $\begin{array}{l}\text { Drost et al. } \\
\text { (2007) }\end{array}$ \\
\hline $\mathrm{CuCl}_{2}$ & L. minor & $\begin{array}{l}\text { FN } \\
\text { FW } \\
\text { DW } \\
\text { CHL } \\
\text { CAR }\end{array}$ & $7 d$ & $\begin{array}{l}330 \\
(236-474) \\
95 \\
(54-150) \\
157 \\
(129-181) \\
136 \\
(121-153) \\
132 \\
(113-154)\end{array}$ & 300 & Steinberg & $100 \pm 15$ & 24 & $25 \pm 1$ & 5.5 & $\begin{array}{l}\text { Naumann et al. } \\
\text { (2007) }\end{array}$ \\
\hline $\mathrm{Cu}$ & L. minor & FA & $4 \mathrm{~d}$ & 470 & 100 & $\begin{array}{l}\text { 1/4 CoÏc\& } \\
\text { Leisant } \\
(1973)\end{array}$ & 101 & 16 & 22 & $6.1 \pm 0.1$ & $\begin{array}{l}\text { Khellaf and } \\
\text { Zerdaoui } \\
\text { (2009) }\end{array}$ \\
\hline $\mathrm{Cu}$ & L. minor & RL & $2 \mathrm{~d}$ & $\begin{array}{l}221.0 \\
(123.9-385.0)\end{array}$ & 3 & Steinberg & $150 \pm 10$ & 24 & $30 \pm 1$ & 7 & This study \\
\hline $\mathrm{CuSO}_{4} \cdot 5 \mathrm{H}_{2} \mathrm{O}$ & L. paucicostata & FN & $7 d$ & $50-100$ & 25 & $\begin{array}{l}\text { Bonner- } \\
\text { Devirian }\end{array}$ & 120 & 24 & $25 \pm 1$ & $4.1-7.1$ & $\begin{array}{l}\text { Nasu and } \\
\text { Kugimoto } \\
\text { (1981) }\end{array}$ \\
\hline $\mathrm{Cu}$ & L. paucicostata & RL & $2 \mathrm{~d}$ & $\begin{array}{l}470.4 \\
(420.8-509.0)\end{array}$ & 3 & Steinberg & $150 \pm 10$ & 24 & $30 \pm 1$ & 7 & This study \\
\hline $\mathrm{Hg}$ & L. gibba & RL & $2 \mathrm{~d}$ & $\begin{array}{l}222.2 \\
(189.4-259.2)\end{array}$ & 3 & Steinberg & $150 \pm 10$ & 24 & $30 \pm 1$ & 7 & This study \\
\hline $\mathrm{HgCl}_{2}$ & L. minor & FN & $5 \mathrm{~d}$ & 2100 & 50 & Hoagland & 40 & 24 & $25 \pm 2$ & & $\begin{array}{l}\text { Subhadra et al. } \\
\text { (1991) }\end{array}$ \\
\hline $\mathrm{HgCl}_{2}$ & L. minor & $\begin{array}{l}\text { FN } \\
\text { FW } \\
\text { DW } \\
\text { CHL } \\
\text { CAR }\end{array}$ & $7 \mathrm{~d}$ & $\begin{array}{l}683 \\
(536-881) \\
191 \\
(155-235) \\
221 \\
(162-301) \\
135 \\
(92-205) \\
123 \\
(75-212)\end{array}$ & 300 & Steinberg & $100 \pm 15$ & 24 & $25 \pm 1$ & 5.5 & $\begin{array}{l}\text { Naumann et al. } \\
\text { (2007) }\end{array}$ \\
\hline $\mathrm{HgCl}_{2}$ & L. minor & FW & $\begin{array}{l}4 \mathrm{~d} \\
7 \mathrm{~d}\end{array}$ & $\begin{array}{l}640 \\
480\end{array}$ & 200 & Jacobs & 40 & 24 & $25 \pm 2$ & 6 & Dirilgen (2011) \\
\hline $\mathrm{Hg}$ & L. minor & RL & $2 \mathrm{~d}$ & $\begin{array}{l}196.2 \\
(93.1-286.8)\end{array}$ & 3 & Steinberg & $150 \pm 10$ & 24 & $30 \pm 1$ & 7 & This study \\
\hline $\mathrm{Hg}$ & L. paucicostata & RL & $2 \mathrm{~d}$ & $\begin{array}{l}106.6 \\
(65.6-330.4)\end{array}$ & 3 & Steinberg & $150 \pm 10$ & 24 & $30 \pm 1$ & 7 & This study \\
\hline
\end{tabular}



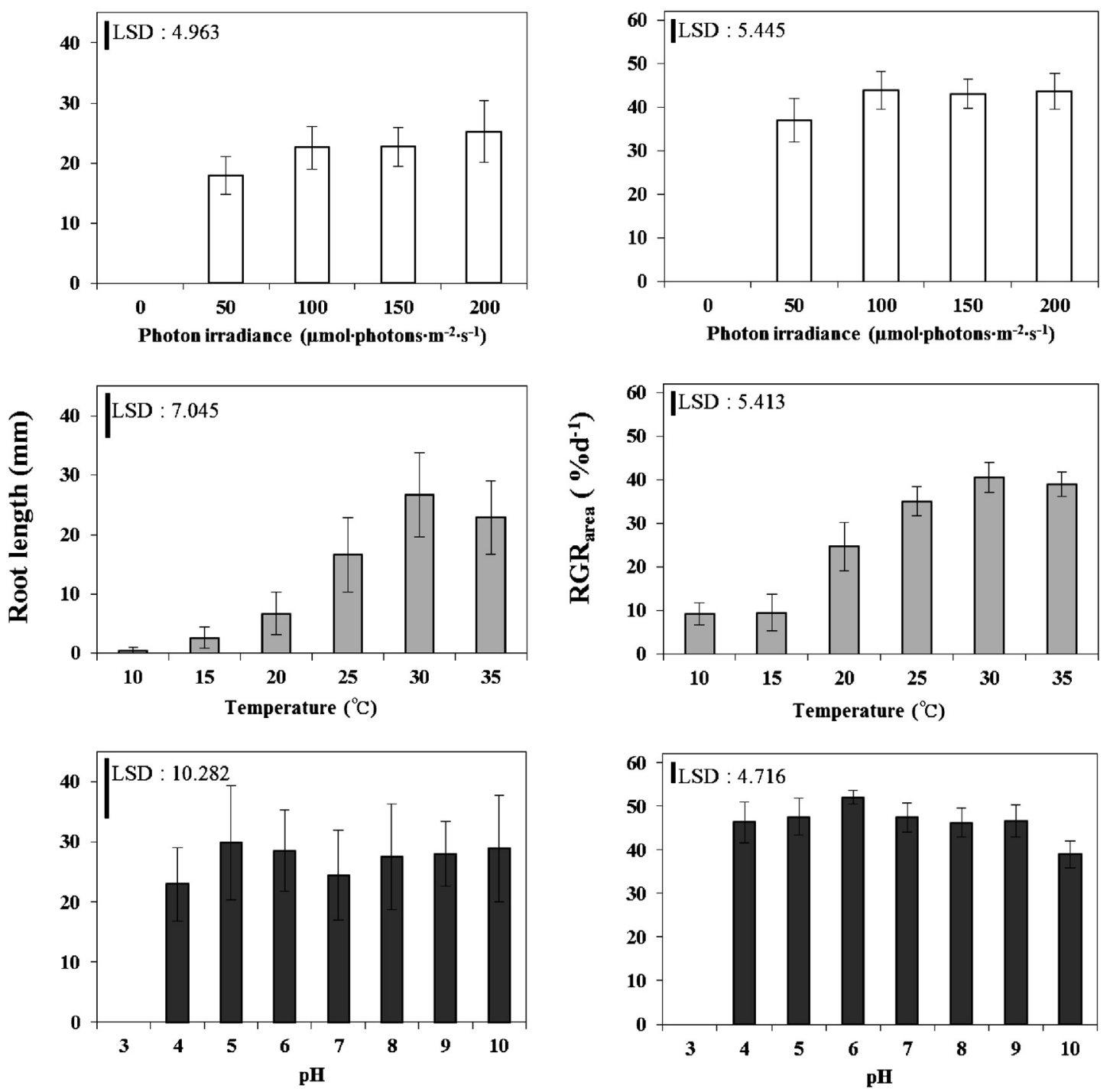

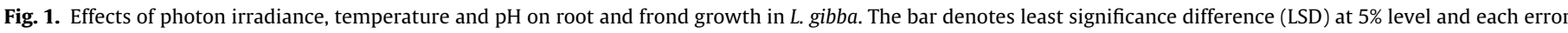
bar indicates $95 \%$ confidence interval $(n=3)$.

reported to be $7^{\circ} \mathrm{C}$ and above $31-35^{\circ} \mathrm{C}$, respectively (Iqbal, 1999). Toxicity of $L$. minor has been shown to be independent of temperatures between 20 and $30^{\circ} \mathrm{C}$ (Nasu and Kugimoto, 1981) although, in other species, toxicity decreases with decreasing temperature (Das et al., 1997). In our study, temperature was set at $30^{\circ} \mathrm{C}$ as this was the temperature for maximal growth of roots and areas of fronds in all three Lemna species. This temperature is at the higher limit of optimal temperatures $\left(22-30^{\circ} \mathrm{C}\right)$ employed for most Lemna tests.

The $\mathrm{pH}$ of the test medium can influence the growth of Lemna plants. For example, the higher the $\mathrm{pH}$ value, the greater the inhibition of frond multiplication in L. paucicostata (Nasu and Kugimoto, 1981). The $\mathrm{pH}$ can also affect the bioavailability of metals by altering speciation, and hence can influence toxicity. The desirable $\mathrm{pH}$ range for Lemna toxicity testing of $\mathrm{Ag}, \mathrm{Cd}, \mathrm{Cr}$ and $\mathrm{Cu}$ was suggested to be 6.1-7.1 (Nasu and Kugimoto, 1981). In the present study, pH was set at $7 \pm 0.2$ since growth of both roots and fronds of three Lemna species was recorded between 4 and 10. Table 1 shows that a wide range of $\mathrm{pH}$ values (4.1-7.5) have generally been employed for Lemna toxicity tests.

From comparisons of the results obtained from the many studies on Lemna spp. it is apparent that there are considerable inconsistencies in the reporting of the sensitivities of the various measured endpoints. For instance, a study of metal toxicity using 5 endpoints (frond number, fresh weight, dry weight, chlorophyll (Chl) and carotenoid (Car) concentrations) in L. minor revealed that Chl and Car were the most sensitive endpoints, while frond number was the least sensitive (Naumann et al., 2007). Contrary to this, Mackenzie et al. (2003) reported that frond number was the most sensitive of the endpoints measured while others (e.g. Teisseire et al., 1998; Lahive et al., 2011), have shown that growth rates, based on changes in biomass, were more sensitive than photosynthetic pigment concentrations. Differences in sensitivity may occur between Lemna species and clones of the same species (Lahive et al., 2011).

Root growth following excision prior to exposure to metals proved to be a sensitive measure of toxic effects. Coefficients of variation of the root test method ranged from 4.22 to $12.82 \%$ for $L$. gibba, from 0.83 to $15.80 \%$ for $L$. minor and from 1.60 to $19.63 \%$ for $L$. paucicostata. CV values of the frond area test method were between 3.64 and $32.12 \%$ (Table 3). These ranges of CV are comparable to those obtained from internationally recognized bioassays carried out on the same metal toxicants. The rank order of metal toxicity, in terms of $\mathrm{EC}_{50} \mathrm{~S}$, was species-specific; for $L$. gibba and L. minor it was $\mathrm{Ag}>\mathrm{Hg}>\mathrm{Cu}>\mathrm{Cd}>\mathrm{Cr}$ whereas it was $\mathrm{Ag}>\mathrm{Cd}>\mathrm{Hg}>\mathrm{Cr}>\mathrm{Cu}$ for L. paucicostata (Table 3). It is notable that $\mathrm{Ag}$ was the most toxic metal for all three species, whereas $\mathrm{Cr}$ was least toxic for $L$. gibba 

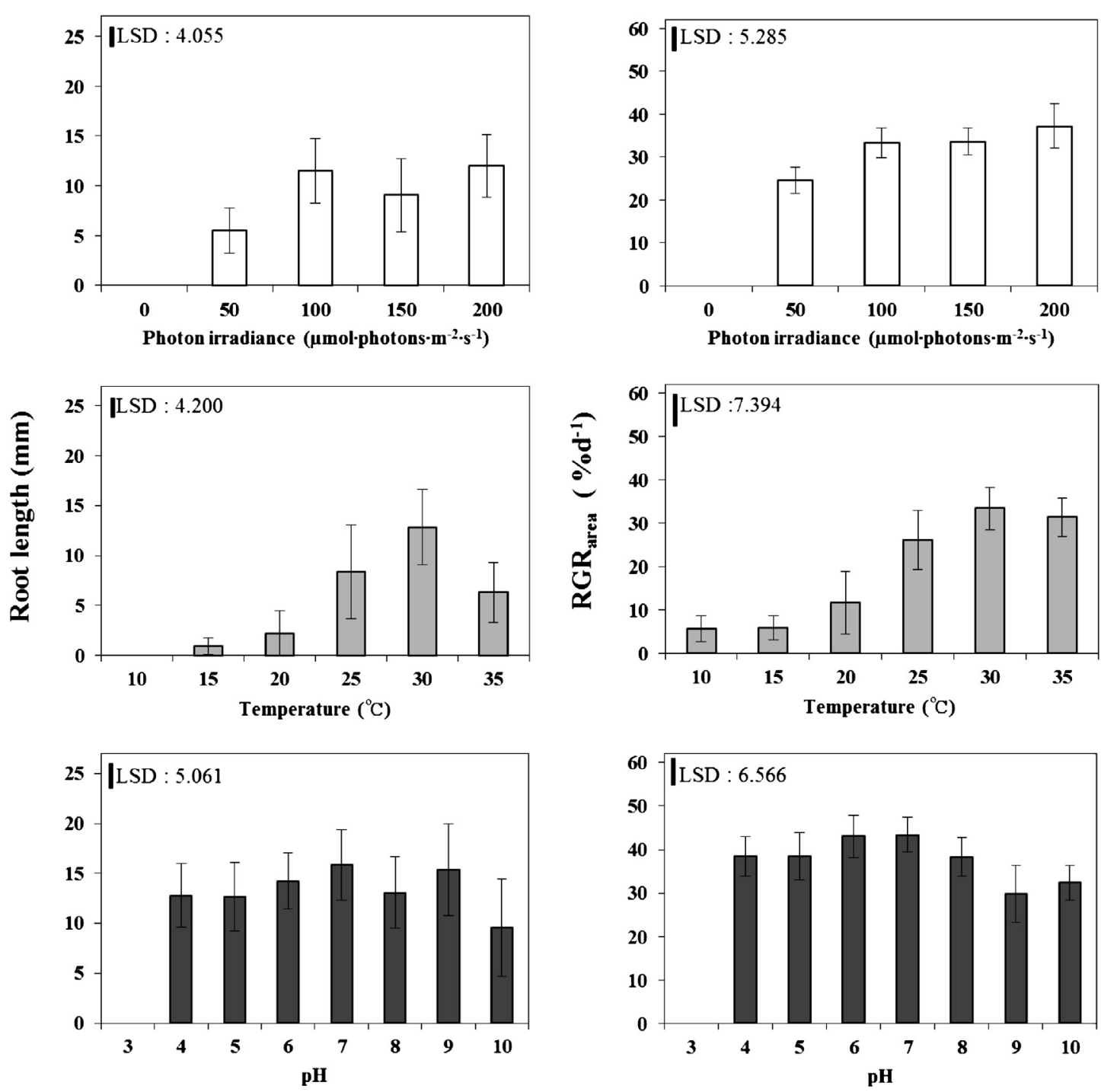

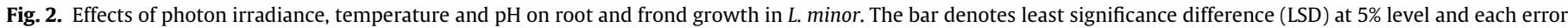
bar indicates $95 \%$ confidence interval $(n=3)$.

and L. minor and $\mathrm{Cu}$ for L. paucicostata. In terms of frond growth, $\mathrm{Ag}$ was also found to be most toxic metal for the three species while the second most toxic metal was either $\mathrm{Hg}$ for $L$. gibba, or Cd for $L$. minor and L. paucicostata.

Silver is a relatively rare element occurring naturally in the earth's crust, and the principal sources of the occurrence of silver and silver compounds are from human uses such as photographic materials, electroplating, electrical conductors, dental alloys, solder and brazing alloys, paints, jewelry, coins, and mirror production. It is also used for seeding clouds, as an antibacterial agent, and has been used for water purification (http://cira.ornl.gov/documents/ SILVER.pdf).

Table 3

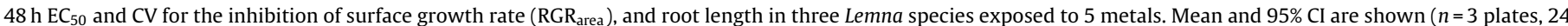
plants per plate with 4 plants per concentration).

\begin{tabular}{|c|c|c|c|c|c|c|c|}
\hline \multirow[t]{2}{*}{ End point } & \multirow{2}{*}{$\begin{array}{l}\text { Heavy } \\
\text { metals }\end{array}$} & \multicolumn{2}{|l|}{ L. gibba } & \multicolumn{2}{|l|}{ L. minor } & \multicolumn{2}{|l|}{ L. paucicostata } \\
\hline & & $\mathrm{EC}_{50}(95 \% \mathrm{CI})$ & $\mathrm{CV}(\%)$ & $\mathrm{EC}_{50}(95 \% \mathrm{CI})$ & $\mathrm{CV}(\%)$ & $\mathrm{EC}_{50}(95 \% \mathrm{CI})$ & $\mathrm{CV}(\%)$ \\
\hline \multirow{5}{*}{$\mathrm{RGR}_{\text {area }}$} & $\mathrm{Ag}$ & $42.6(36.7-55.6)$ & 9.71 & $50.8(37.6-60.1)$ & 8.30 & $74.5(66.4-81.1)$ & 19.35 \\
\hline & $\mathrm{Cd}$ & $361.6(323.2-410.4)$ & 17.49 & $516.6(314.8-620.9)$ & 5.51 & $403.6(381.7-429.1)$ & 32.12 \\
\hline & $\mathrm{Cr}^{6+}$ & $>3000$ & - & $>3000$ & - & $>2500$ & - \\
\hline & $\mathrm{Cu}$ & $486.2(421.2-599.3)$ & 10.42 & 1774.9 & - & 2448.7000 & - \\
\hline & $\mathrm{Hg}$ & $210.6(160.5-290.2)$ & 3.64 & $>1000$ & - & $913.5(808.3-1036.9)$ & 16.71 \\
\hline \multirow{5}{*}{ Root length } & $\mathrm{Ag}$ & $27.6(21.3-34.2)$ & 7.25 & $5.3(2.6-23.1)$ & 0.83 & $37.6(28.6-44.3)$ & 10.07 \\
\hline & $\mathrm{Cd}$ & $363.5(257.4-407.6)$ & 7.91 & $226.4(200.7-248.5)$ & 15.80 & $77.0(61.1-118.1)$ & 5.10 \\
\hline & $\mathrm{Cr}^{6+}$ & $1148.3(267.1-1403.6)$ & 4.22 & $341.8(163.0-559.7)$ & 3.38 & $237.9(195.0-280.5)$ & 10.99 \\
\hline & $\mathrm{Cu}$ & $310(236.7-391.3)$ & 6.91 & $221(123.9-385.0)$ & 3.47 & $470.4(420.8-509.0)$ & 19.63 \\
\hline & $\mathrm{Hg}$ & $222.2(189.4-259.2)$ & 12.82 & $196.2(93.1-286.8)$ & 3.96 & $106.6(65.6-330.4)$ & 1.60 \\
\hline
\end{tabular}



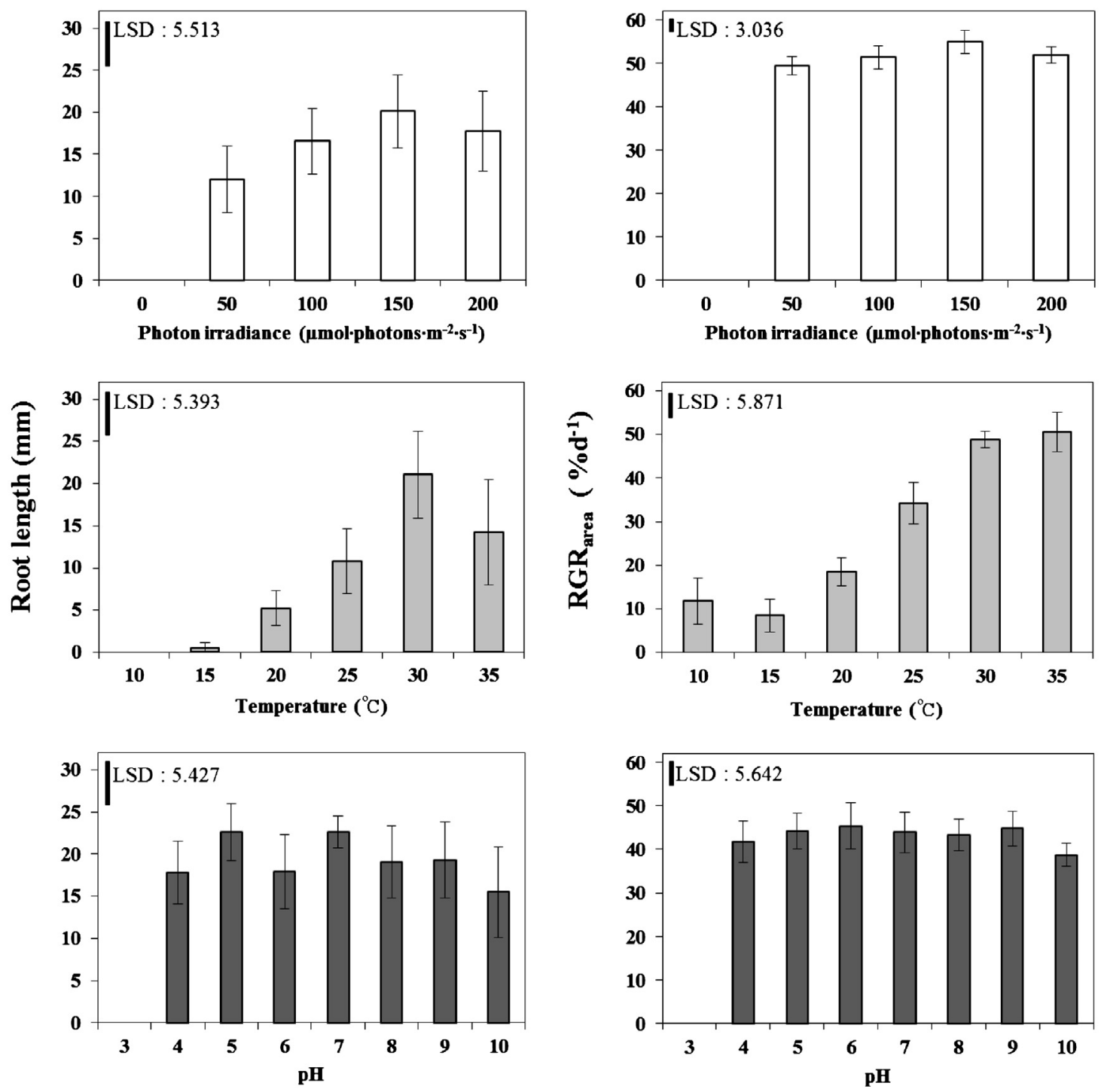

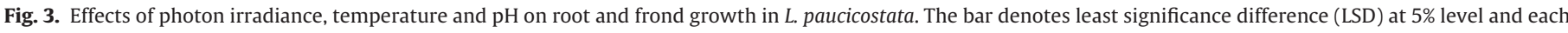
error bar indicates $95 \%$ confidence interval $(n=3)$.

Even in its bulk form, silver is reported to be extremely toxic to plants and algae, although the mode of action remains poorly understood (Eisler, 1996). Silver, or silver nanoparticles, can cause abscission of fronds and inhibit multiplication of fronds and frond growth of Lemna (Nasu and Kugimoto, 1981; Naumann et al., 2007; Topp et al., 2011). In L. minor derived $\mathrm{EC}_{50}$ values, from growth rates, based on frond number and biomass, and photosynthetic pigment concentrations, varied between 24 and $81 \mu \mathrm{g} \mathrm{L}^{-1}$ (Naumann et al., 2007), while Topp et al. (2011) reported that $\mathrm{EC}_{50}$ s, for frond abscission differed between clones of the species e.g. values between 51 and $1296 \mu \mathrm{g} \mathrm{L}^{-1}$ for clone 7868 (from Ireland) and clone 7022 (from Spain), respectively (Table 2). Based on root growth measurements the most sensitive species to $\mathrm{Ag}$ was L. minor $\left(\mathrm{EC}_{50}\right.$ of $\left.5.3 \mu \mathrm{g} \mathrm{L}^{-1}\right)$ followed by L. gibba $\left(27.6 \mu \mathrm{g} \mathrm{L}^{-1}\right)$ and $L$. paucicostata $\left(37.6 \mu \mathrm{g} \mathrm{L}^{-1}\right.$ ) (Table 3). For frond growth (area) $\mathrm{EC}_{50}$ values were $42.6 \mu \mathrm{g} \mathrm{L}^{-1}$ for $L$. gibba, $50 \mu \mathrm{g} \mathrm{L}^{-1}$ for $L$. minor and $74.5 \mu \mathrm{g} \mathrm{L}^{-1}$ for $L$. paucicostata. Therefore, the sensitivity of root growth of $L$. minor to Ag was ca. 5-245 times higher than that of other more conventional endpoints (Table 2). The sensitivity of root growth in $L$. gibba and L. paucicostata to $\mathrm{Ag}$ was also similar to that of the most sensitive endpoints that have so far been reported. Generally, responses of roots to Ag were more sensitive than fronds.
Cd occurs in natural and waste waters and originates from many industrial sources such as processing, smelting and mining ores, reclamation of scrap metals, incineration for disposal of waste products, run-off carrying fertilizers and fungicides etc. (Schmitz, 1996). Cadmium poses toxic effects on plants by interfering with chlorophyll pigment formation, enzymatic activity, and $\mathrm{CO}_{2}$ fixation, leading to an overall reduction in growth (Fodor, 2002). In Lemna, Cd can reduce cellular protein, carbohydrate and, chlorophyll contents, some antioxidant activity and to inhibit multiplication of frond number, area and biomass (Nasu and Kugimoto, 1981; Smith and Kwan, 1989; Mohan and Hosetti, 1997; Naumann et al., 2007; Megateli et al., 2009; Malec et al., 2010). In the present study, the $\mathrm{EC}_{50}$ of $\mathrm{Cd}$ toxicity, based on root growth measurements, was significantly lower $(P<0.05)$ in $L$. paucicostata $\left(77 \mu \mathrm{g} \mathrm{L}^{-1}\right)$ than in $L$. gibba (363.5 $\left.\mu \mathrm{g} \mathrm{L}^{-1}\right)$ or $L$. minor $\left(226.4 \mu \mathrm{g} \mathrm{L}^{-1}\right.$ ) (Table 3$)$. In contrast, effects of $\mathrm{Cd}$ on frond growth did not differ significantly between the species. The $\mathrm{EC}_{50}$ values obtained for $L$. minor and $L$. paucicostata were lower than the corresponding values for root growth. In the wild-type L. paucicostata colony break-up was observed more frequently on exposure to $\mathrm{Cd}$ than to the other 6 metals tested (Li and Xiong, 2004). When compared with other published reports (Table 2), Cd toxicity to $L$. paucicostata, based on $\mathrm{EC}_{50}$ 
Table 4

Comparison of 3 internationally standardized Lemna test methods with the root test method developed in this study.

\begin{tabular}{|c|c|c|c|c|}
\hline & ISO & OECD & EPA & This study \\
\hline Test species & Lemna minor & L. gibba, L. minor & L. gibba G3, L. minor & L. gibba, L. minor, L. paucicostata \\
\hline Test duration & 7 days & 7 days & 7 days & 2 days \\
\hline Temperature $\left({ }^{\circ} \mathrm{C}\right)$ & $24 \pm 2{ }^{\circ} \mathrm{C}$ & $24 \pm 2{ }^{\circ} \mathrm{C}$ & $25 \pm 2{ }^{\circ} \mathrm{C}$ & $30 \pm 1^{\circ} \mathrm{C}$ \\
\hline Salinity & Fresh water & Fresh water & Fresh water & Fresh water \\
\hline Light intensity & $85-135 \mu \mathrm{mol}$ photons $\mathrm{m}^{-2} \mathrm{~s}^{-1}$ & $6500-10,000$ lux & 4200-6700 lux & $150 \mu \mathrm{mol}$ photons $\mathrm{m}^{-2} \mathrm{~s}^{-1}$ \\
\hline Photoperiod & Continuous light & Continuous light & Continuous light & Continuous light \\
\hline Test vessel type & Beaker & Flasks, Petri dish & Beaker, flask & 24-well plate \\
\hline Medium & Steinberg medium & $\begin{array}{l}\text { Swedish Standard (SIS) lemna } \\
\text { medium for } L \text {. minor } 20 \times \text { AAP } \\
\text { growth medium for } L \text {. gibba }\end{array}$ & $\begin{array}{l}\text { M-Hoagland's medium or } \\
20 \times \text {-AAP nutrient medium }\end{array}$ & Steinberg medium \\
\hline Test solution volume & $100 \mathrm{ml}$ (minimum) & $100 \mathrm{ml}$ (minimum) & $150 \mathrm{ml}$ & $3 \mathrm{ml}$ \\
\hline Test solution $\mathrm{pH}$ & 5.5 & $6.5 \pm 0.2$ & $7.5 \pm 0.1$ & $7 \pm 0.2$ \\
\hline Test organism size & $\begin{array}{l}10-16 \text { fronds per test vessel ( } 2 \\
\text { or } 3 \text { fronds per colony) }\end{array}$ & 9-12 fronds per test vessel & $12-16$ fronds per test vessel & $\begin{array}{l}1 \text { colony per test vessel ( } 2 \\
\text { fronds per colony) }\end{array}$ \\
\hline Endpoint & $\begin{array}{l}\text { Growth rate (frond number, } \\
\text { frond area, dry weight, } \\
\text { chlorophyll contents) }\end{array}$ & $\begin{array}{l}\text { Average specific growth rate, } \\
\text { final biomass, area under the } \\
\text { growth curve }\end{array}$ & $\begin{array}{l}\text { Total frond number, growth } \\
\text { rate (as number of fronds per } \\
\text { day), mortality (\% of dead } \\
\text { fronds to total number of } \\
\text { fronds) and dry weight, } \\
\text { chlorophyll and pheophytin } \\
\text { pigment analyses }\end{array}$ & Root length \\
\hline Test type & Static none-renewal & Static none-renewal & Static none-renewal & Static none-renewal \\
\hline Condition & Axenic culture & Axenic culture & Axenic culture & Non-axenic culture \\
\hline
\end{tabular}

values derived from root and frond growth, was similar to or greater than those from measurements of biomass (110-241 $\left.\mu \mathrm{g} \mathrm{L}^{-1}\right)$ and frond number (200-2500 $\mu \mathrm{g} \mathrm{L}^{-1}$ ) in L. minor (Drost et al., 2007; Khellaf and Zerdaoui, 2009). The present results appear to agree with the previous findings that $L$. paucicostata may be a particularly sensitive species to $\mathrm{Cd}$.

Chromium has multifarious industrial uses for leather processing, the production of refractory steel, drilling muds, electroplating cleaning agents, catalytic manufacture, chromic acid and specialty chemicals, metal plating, cooling tower water treatment, and wood preservation (Shanker et al., 2005). Chromium compounds are reported to be highly toxic to plants, being detrimental to physiological mechanisms including $\mathrm{CO}_{2}$ fixation, electron transport, photophosphorylation, chlorophyll biosynthesis, enzyme activities, leading to negative impacts on their essential life processes such as growth and development (Vajpayee et al., 2000; Shanker et al., 2005). Plants of the Lemnaceae appear to be relatively more tolerant of $\mathrm{Cr}$ than other metals (Landolt and Kandeler, 1987). The maximal $\mathrm{EC}_{50} \mathrm{~S}$ of $\mathrm{Cr}$ toxicity recorded (Table 2) are $1148.3 \mu \mathrm{g} \mathrm{L}^{-1}$ for $L$. gibba, $341.8 \mu \mathrm{g} \mathrm{L}^{-1}$ for L. minor, and $237.9 \mu \mathrm{g} \mathrm{L}^{-1}$ for L. paucicostata, and these values are, for example, 6-10 times higher than the $\mathrm{EC}_{50} \mathrm{~S}$ of $\mathrm{Ag}$ toxicity for the corresponding species (Table 2). The $\mathrm{EC}_{50}$ values derived from frond growth were significantly higher than those for root growth. The newly developed root system appears to be more sensitive to $\mathrm{Cr}$ exposure than fronds as is evident from a comparison of $\mathrm{EC}_{50} \mathrm{~s}$ from measurements of root growth with more traditional endpoints (Table 2); for $L$. gibba, values are $1148.3 \mu \mathrm{g} \mathrm{L}^{-1}$ vs $48,000-49,000 \mu \mathrm{gL}^{-1}$ for roots and fronds respectively, $341.8 \mu \mathrm{g} \mathrm{L}^{-1}$ vs $584-40,000 \mu \mathrm{g} \mathrm{L}^{-1}$ for $L$. minor and $237.9 \mu \mathrm{g} \mathrm{L}^{-1}$ vs $1000 \mu \mathrm{g} \mathrm{L}^{-1}$ for L. paucicostata (Tables 2 and 3).

There are various anthropogenic sources of $\mathrm{Cu}$ entering aquatic ecosystems including mine drainage, industrial, domestic, agricultural waste, agrochemicals and antifouling paints (Ma et al., 2003; Andrade et al., 2004). Although $\mathrm{Cu}$ is an essential micronutrient and structural and catalytic component for metabolic pathway in plants, exposure to $\mathrm{Cu}$ at concentrations beyond a certain threshold induces phytotoxic symptoms in Lemna, including the inhibition of growth, photosynthesis, respiration, pigment synthesis and enzymatic activity (Teisseire and Vernet, 2005; Kanoun-Boule et al., 2009; Khellaf and Zerdaoui, 2010).
The present study shows that $\mathrm{Cu}$ was significantly $(P<0.05)$ more toxic to $L$. minor $\left(\mathrm{EC}_{50} 221.0 \mu \mathrm{g} \mathrm{L}^{-1}\right)$ than both $L$. gibba $\left(310.0 \mu \mathrm{g} \mathrm{L}^{-1}\right)$ and $L$. paucicostata $\left(470.4 \mu \mathrm{g} \mathrm{L}^{-1}\right)$ (Table 2). The $E_{50}$ values obtained for $L$. gibba and $L$. minor based on root growth measurements are similar to those (95-1100 $\left.\mu \mathrm{g} \mathrm{L}^{-1}\right)$ from measurements of frond number, frond weight and pigment concentrations (Table 2). In the case of $L$. paucicostata, however, the EC values are greater in $\operatorname{root}\left(470.4 \mu \mathrm{g} \mathrm{L}^{-1}\right)$ than in frond growth (50-100 $\mu \mathrm{g} \mathrm{L}^{-1}$ ) (Table 2). A direct comparison of $\mathrm{EC}_{50} \mathrm{~S}$ from measurements of root with frond growth showed that root was significantly $(P>0.05)$ more sensitive to $\mathrm{Cu}$ than frond for all three Lemna species (Table 3 ).

Anthropogenic sources of $\mathrm{Hg}$ come mostly from stationary coal combustion, gold and non-ferrous metal prospecting, cement production, waste disposal, human crematoria, caustic soda production, pig iron and steel production, mercury production (mostly for batteries), and incineration of biomass (Pacyna et al., 2006; Dirilgen, 2011).

$\mathrm{Hg}$ is known to be one of the most toxic metals because of its mobility, bioaccumulation and methylation process. In plants, $\mathrm{Hg}$ has harmful effects on various physiological and biochemical processes, such as vegetative growth, germination, oxygen evolution, chlorophyll $a$ fluorescence, pigmentation, and enzymatic activities (Patra and Sharma, 2000). In the present study, there was no significant difference between species in the sensitivity of root growth to $\mathrm{Hg}$; the $\mathrm{EC}_{50}$ values were $222.2 \mu \mathrm{g} \mathrm{L}^{-1}$ for L. gibba, $196.2 \mu \mathrm{g} \mathrm{L}^{-1}$ for L. minor and $106.6 \mu \mathrm{g} \mathrm{L}^{-1}$ for L. paucicostata (Table 3). Root length was a more sensitive endpoint to $\mathrm{Hg}$ than frond area for $L$. minor and $L$. paucicostata but was the same for $L$. gibba. More generally, the $\mathrm{EC}_{50}$ s obtained from root growth measurements were similar to or higher (68.3-106.6 $\mu \mathrm{g} \mathrm{L}^{-1}$ ) than those based on the measurements of frond parameters (Table 2 ).

\section{Conclusions}

The measurements of root re-growth following their excision showed species-specific differences in sensitivity to five important metal species ( $\mathrm{Ag}, \mathrm{Cd}, \mathrm{Cr}, \mathrm{Cu}$ and $\mathrm{Hg}$ ) in three Lemna species ( $L$. minor, L. gibba, L. paucicostata). TheEC $\mathrm{C}_{50}$ values indicated that root growth was a more sensitive measure of toxicity $(\mathrm{Ag}, \mathrm{Cd}, \mathrm{Cr})$ or of similar sensitivity ( $\mathrm{Cu}, \mathrm{Hg}$ ) compared with the more commonly 
measured parameters on fronds. Table 4 compares the present Lemna root test method with 3 internationally standardized methods (ISO, OCED and US EPA). The root growth bioassay differs in several key respects: the test can be completed in only $48 \mathrm{~h}$, the test vessel is a 24-well cell plate, the required volume of test water samples is $3 \mathrm{ml}$, and axenic cultures, which are costly and difficult to maintain, are not required. Furthermore, the technique of excising roots prior to exposure means that there is no requirement to pre-select roots of uniform length and it reduces the amount of handing of these fragile roots.

The results of this investigation clearly show that Lemna root method is a simple, rapid, cost-effective and precise bioassay that can be used to assess the toxic risks of metals. It has practical applications for monitoring municipal and industrial waste waters where metals are common constituents. Further investigations are currently underway to evaluate the sensitivity of the method to a wider range of toxic chemicals.

\section{Acknowledgements}

This research was supported by the Korea Ministry of Environment (Grant no. 401-111-001) and by a research grant from Incheon National University, SDG.

\section{References}

Andrade, L.R., Farina, M., Amado Filho, G.M., 2004. Effects of copper on Enteromorpha flexuosa (Chlorophyta) in vitro. Ecotoxicol. Environ. Saf. 58, 117-125.

Christen, O., Theuer, C., 1996. Sensitivity of Lemna-bioassay interacts with stockculture period. J. Chem. Ecol. 22, 1177-1186.

Cowgill, U.M., Milazzo, D.P., Landenberger, B.D., 1991. The sensitivity of Lemna gibba G 3 and four clones of Lemna minor to eight common chemicals using a 7-day test. Res. J. Water Pollut. 63, 991-998.

Das, P., Samantaray, S., Rout, G.R., 1997. Studies on cadmium toxicity in plants: a review. Environ. Pollut. 98, 29-36.

Davis, J.A., 1981. Comparison of Static-replacement and Flow Through Bioassays Using Duckweed, Lemna gibba G3. EPA 560/6-81-003. United States Environmental Protection Agency, Washington, DC.

Dirilgen, N., 2011. Mercury and lead: assessing the toxic effects on growth and metal accumulation by Lemna minor. Ecotoxicol. Environ. Saf. 74, 48-54.

Drost, W., Matzke, M., Backhaus, T., 2007. Heavy metal toxicity to Lemna minor: studies on the time dependence of growth inhibition and the recovery after exposure. Chemosphere 67, 36-43.

Eisler, R., 1996. Silver Hazards to Fish, Wildlife and Invertebrates: a Synoptic Review. US Department of the Interior, National Biological Service, Washington, DC, USA, Biological Report 32.

Eullaffroy, P., Vernet, G., 2003. The F684/F735 chlorophyll fluorescence ratio: a potential tool for rapid detection and determination of herbicide phytotoxicity in algae. Water Res. 37, 1983-1990.

Fodor, F., 2002. Physiological responses of vascular plants to heavy metals. In: Prasad, M.N.V., Strzałka, K. (Eds.), Physiology and Biochemistry of Metal Toxicity and Tolerance in Plants. Kluwer Academic Publishers, Amsterdam, pp. 149-177.

Greenberg, B.M., Huang, X.D., Dixon, D.G., 1992. Applications of the aquatic higherplant Lemna gibba for ecotoxicological assessment. J. Aquat. Ecosyst. Health 1, 147-155.

Gubbins, E.J., Batty, L.C., Lead, J.R., 2011. Phytotoxicity of silver nanoparticles to Lemna minor L. Environ. Pollut. 159, 1551-1559.

Hillman, W.S., 1961. The Lemnaceae or duckweeds: a review of the descriptive and experimental literature. Bot. Rev. 27, 221-287.

ISO, 2005. Water Quality - Determination of the Toxic Effect of Water Constituents and Waste to Duckweed (Lemna minor) - Duckweed Growth Inhibition Test. International Organization for Standardization, Geneva, Switzerland.

Iqbal, S., 1999. Duckweed Aquaculture: Potentials, Possibilities and Limitations for Combined Wastewater Treatment and Animal Feed Production in Developing Countries, SANDEC Report No. 6/99, EAWAG.

Kanoun-Boule, M., Vicente, J.A.F., Nabais, C., Prasad, M.N.V., Freitas, H., 2009. Ecophysiological tolerance of duckweeds exposed to copper. Aquat. Toxicol. 91, $1-9$.

Khellaf, N., Zerdaoui, M., 2009. Growth response of the duckweed Lemna minor to heavy metal pollution. Iran J. Environ. Health Sci. Eng. 6, 161-166.

Khellaf, N., Zerdaoui, M., 2010. Growth response of the duckweed Lemna gibba L. to copper and nickel phytoaccumulation. Ecotoxicology 19, 1363-1368.
Kumar, K.S., Han, T., 2010. Physiological response of Lemna species to herbicides and its probable use in toxicity testing. Toxicol. Environ. Health Sci. 2, 39-49.

Lahive, E., O'Halloran, J., Jansen, M.A.K., 2011. Differential sensitivity of four Lemnaceae species to zinc sulphate. Environ. Exp. Bot. 71, 25-33.

Landolt, E., Kandeler, R., 1987. Biosystematic investigations in the family of duckweeds (Lemnaceae). Veroff. Geobot. Inst. ETH Zurich 2, 42-43.

Li, T., Xiong, Z.-T., 2004. A novel response of wild-type duckweed (Lemna paucicostata Hegelm.) to heavy metals. Environ. Toxicol. 19, 95-102.

Ma, M., Zhu, W., Wang, Z., Witkamp, G., 2003. Accumulation, assimilation and growth inhibition of copper on freshwater alga (Scenedesmus subspicatus 86.81 SAG) in the presence of EDTA and fulvic acid. Aquat. Toxicol. 63, 221-228.

Malec, P., Maleva, M.G., Prasad, M.N.V., Strzałka, K., 2010. Responses of Lemna trisulca L. (duckweed) exposed to low doses of cadmium: thiols, metal binding complexes, and photosynthetic pigments as sensitive biomarkers of ecotoxicity. Protoplasma 240, 69-74.

Mangi, J., Schmidt, K., Pankow, J., Gaines, L., Turner, P., 1978. Effects of chromium on some aquatic plants. Environ. Pollut. 16, 285-291.

Mackenzie, S.M., Waite, S., Metcalfe, D.J., Joyce, C.B., 2003. Landfill leachate ecotoxicity experiments using Lemna minor. Water Air Soil Pollut. Focus 3, 171-179.

Megateli, S., Semsari, S., Couderchet, M., 2009. Toxicity and removal of heavy metals (cadmium, copper, and zinc) by Lemna gibba. Ecotoxicol. Environ. Saf. 72, 1774-1780

Mohan, A.S., Hosetti, B.G.B., 1997. Potential phytotoxicity of lead and cadmium to Lemna minor growth in sewage stabilization ponds. Environ. Pollut. 98, 233-238.

Munzuroglu, O., Geckil, H., 2002. Effects of metals on seed germination, root elongation, and coleoptile and hypocotyl growth in Triticum aestivum and Cucumis sativus. Arch. Environ. Contam. Toxicol. 43, 203-213.

Nasu, Y., Kugimoto, M., 1981. Lemna (duckweed) as an indicator of water pollution. I. The sensitivity of Lemna paucicostata to heavy metals. Arch. Environ. Contam. Toxicol. 10, 159-169.

Naumann, B., Eberiusb, M., Appenrotha, K., 2007. Growth rate based dose-response relationships and EC-values of ten heavy metals using the duckweed growth inhibition test (ISO 20079) with Lemna minor L. clone St. J. Plant Physiol. 164, $1656-1664$.

OECD, 2002. Guidelines for the Testing of Chemicals. Lemna sp. Growth Inhibition Test, Draft Guideline 221

Pacyna, E.G., Pacyna, J.M., Steenhuisen, F., Wilson, S., 2006. Global anthropogenic mercury emission inventory for 2000. Atmos. Environ. 40, 4048-4063.

Panda, S.K., Upadhyay, R.K., 2003. Salt stress injury induces oxidative alteration and antioxidative defence in the roots of Lemna minor. Biol. Plant 48, 249-253.

Patra, M., Sharma, A., 2000. Mercury toxicity in plants. Bot. Rev. 66, 379-422.

Prasad, M.N.V., Malec, P., Waloszek, A., Bojko, M., Strzal-ka, K., 2001. Physiological responses of Lemna trisulca L. (duckweed) to cadmium and copper bioaccumulation. Plant Sci. 161, 881-889.

Schmitz, R.J., 1996. Introduction to Water Pollution Biology. Gulf Publishing, Houston.

Shanker, A.K., Cervantes, C., Loza-Tavera, H., Avudainayagam, S., 2005. Chromium toxicity in plants. Environ. Int. 31, 739-753.

Smith, S., Kwan, M.K.H., 1989. Use of aquatic macrophytes as a bioassay method to assess relative toxicity, uptake kinetics and accumulated forms of trace metals. Hydrobiologia 188/189, 345-351.

Steinberg, R., 1946. Mineral requirement of Lemna minor. Plant Physiol. 21, 42-48.

Subhadra, A.V., Nanda, A.K., Behera, P.K., Panda, B.P., 1991. Acceleration of catalase and peroxidase activities in Lemna minor L. and Allium cepa L. in response to low levels of aquatic mercury. Environ. Pollut. 69, 169-179.

Teisseire, H., Couderchet, M., Vernet, G., 1998. Toxic responses and catalase activity of Lemna minor exposed to folpet, copper, and their combination. Ecotoxicol. Environ. Saf. 40, 194-200.

Teisseire, H., Vernet, G., 2000. Ascorbate and glutathione contents in duckweed Lemna minor, as biomarkers of the stress generated by copper, folpet and diuron. Biomarkers 5, 263-273.

Teisseire, H., Vernet, G., 2005. Copper induced changes in antioxidant enzymes activities in fronds of duckweed (Lemna minor). Plant Sci. 153, 65-72.

Topp, C., Henke, R., Keresztes, A., Fischer, W., Eberius, M., Appenroth, K.J., 2011. A novel mechanism of abscission in fronds of Lemna minor L. and the effect of silver ions. Plant Biol. 13, 517-523.

USEPA, 1996. Aquatic Plant Toxicity Test Using Lemna spp. United States Environmental Protection Agency, EPA 712-C-96-156.

Vajpayee, P., Tripati, R.D., Rai, U.N., Ali, M.B., Singh, S.N., 2000. Chromium accumulation reduces chlorophyll biosynthesis, nitrate reductase activity and protein content of Nymphaea alba. Chemosphere 41, 1075-1082.

Wang, W., 1986. Toxicity tests of aquatic pollutants by using common duckweed. Environ. Pollut. Ser. B: Chem. Phys. 11, 1-14.

Wang, W., 1991. Literature review on higher plants for toxicity testing. Water Air Soil Pollut. 59, 381-400

Wang, W., Williams, J.M., 1990. The use of phytotoxicity tests (common duckweed cabbage, and millet) for determining effluent toxicity. Environ. Monit. Assess. $14,45-58$.

Zhou, X., Li, Q., Arita, A., Sun, H., Costa, M., 2009. Effects of nickel, chromate, and arsenite on histone 3 lysine methylation. Toxicol. Appl. Pharmacol. 236, 78-84 